\title{
EDITORIAL
}

\section{Recent Advances in Artificial Intelligence}

Welcome to the special issue the Journal of Intelligent Systems on "Recent Advances in Artificial Intelligence". This special issue consists of five different papers on $\mathrm{AI}$ and its applications. Each paper was peer reviewed by two independent experts.

The paper COBBER: Ontology Based Model for Human Centered Computing by Héctor Gómez-Gauchía, Belén Díaz-Agudo and Pedro González-Calero, all from the Departamento de Ingeniería Software e Inteligencia Artificial, GAIA Research Group, Facultad de Informatica, Universidad Complutense, Madrid, Spain. In this paper, the authors present a domain-independent approach for supporting human centered computing issues. This approach considers psychological and cognitive issues of users when interacting with the model.

The next paper, An Influence Diagram Based Approach for Estimation of Staff Training in Software Industry, is authored by Kawal Jeet, Vijay Kumar Mago, Department of Computer Science, DAV College, Jalandhar, India, Bhanu Prasad, Department of Computer and Information Sciences, Florida A\&M University, Tallahassee, FL, USA, and Rajinder Singh Minhas, Department of Computer Science, MLUDAV College, Phagwara, India. The authors present a system based on influence diagrams to assess the cost of staff training in software industry.

The next paper, Humor in the Blogosphere: First Clues for a Verbal Humor Taxonomy, is authored by Antonio Reyes, Paolo Rosso, and Davide Buscaldi, all from Natural Language Engineering Lab-EliRF, Departamento de Sistemas Informáticos y Computación, Universidad Politécnica de Valencia, Spain. The authors investigated the features that define a corpus of humorous data, for one-liners, to assess their suitability for building a verbal humor taxonomy.

The next paper of the special issue is, A Website Recommender System based on Analysis of User's Access Log. This paper is authored by Punam Bedi, Bhavna Gupta. Jyotsna Talreja, and Mansi Sood, all from the Department of Computer Science, University of Delhi, India; and Harmeet Kaur, from Hans Raj College, University of Delhi, India. The authors present a website recommendation system that is based on web mining techniques.

The final paper of this special issue is authored by Ugo Galassi from Dipartimento di Informatica, Università Amedeo Avogadro, Alessandria, Italy; Gueanel Cabanes from LIPN-CNRS UMR 7030, Université Paris XIII, Paris, 
France; and Dominique Fresneau from LEEC, Université Paris XIII, Villetaneuse, Paris, France. In their paper, Modeling Evolving Behaniors in Ant Colonies, the authors presented an approach for analyzing the activity logs from an ant colony and used a hidden Markov Model to build a model for the activities.

\section{Dr. Zoran Majkic, Ph.D., Guest Editor}

ETF, Applied Mathematics Department

University of Belgrade, Serbia 